\title{
An XMM-Newton study of the supernova remnant G296.7-0.9
}

\author{
R. H. H. Huang ${ }^{1}$, C. Y. Hui ${ }^{2}$, L. Trepl ${ }^{3}$ and A. K. H. Kong ${ }^{1}$ \\ ${ }^{1}$ Institute of Astronomy and Department of Physics, National Tsing Hua University, \\ Hsinchu, Taiwan \\ email: rhuang@phys.nthu.edu.tw \\ ${ }^{2}$ Department of Astronomy and Space Science, Chungnam National University, Daejeon, Korea \\ ${ }^{3}$ Astrophysikalisches Institut und Universitäts-Sternwarte, Universität Jena, Jena, Germany
}

\begin{abstract}
We report on XMM-Newton observations of the Galactic supernova remnant G296.70.9. A detailed spectro-imaging X-ray study of G296.7-0.9 was performed. We detected an incomplete shell-like X-ray structure which is located near the boundary of the radio emission at a frequency of $843 \mathrm{MHz}$. The X-ray spectrum can be best described by an absorbed ionization plasma model accompanied with metallic emission lines, which suggests the plasma is shock heated. No promising compact stellar remnant associated with G296.7-0.9 was found. No Gamma-ray emission of G296.7-0.9 from Fermi-LAT telescope was detected in our study.
\end{abstract}

Keywords. ISM: supernova remnants, X-rays: individual (G296.7-0.9)

\section{Introduction}

Supernovae (SNe) and their remnants (SNRs) play a crucial role in the heating and chemical evolution of galaxies. To develop a more general understanding of SN explosions, the interaction between ejected materials and the interstellar medium (ISM), particle acceleration in SNRs, and the effect on their galaxy is indispensable. Until now there are 302 SNRs that have been uncovered in the Milky Way: 274 objects recorded in Green's catalog (2009) plus 28 objects reported in Ferrand \& Safi-Harb (2012). Among those Galactic SNRs, only $\sim 100$ of them have been detected in X-rayst. Therefore, to enlarge the sample of X-ray detected SNRs would be valuable.

G296.7-0.9 was initially classified as HII region G296.593-0.975 based on its association with radio recombination lines and an apparently thermal spectrum (Manchester 1969, Caswell \& Haynes 1987, Kuchar \& Clark 1997, and references therein). However, Schaudel (2003) suggested that G296.7-0.9 is a promising SNR candidate based on its X-ray and radio properties. Its incomplete shell-like morphology and thermal X-ray spectra which can be described well by the Raymond-Smith and the thermal bremsstrahlung models with temperatures of $k T=0.22 \pm 0.13 \mathrm{keV}$ and $k T=0.33 \pm 0.15 \mathrm{keV}$ provide a strong support for the SNR interpretation. A subsequent multiwavelength study reported by Robbins et al. (2012) confirms that G296.7-0.9 is a Galactic SNR.

In this proceeding, we present a detailed X-ray study of the newly identified Galactic SNR G296.7-0.9 using XMM-Newton observations.

\section{Observation \& Data Analysis}

G296.7-0.9 was observed with XMM-Newton on June 28, 2011 for a 13 ks total exposure. In this observation, the EPIC MOS1/2 and PN instruments were operated in

$\dagger$ http://www.physics.umanitoba.ca/snr/SNRcat/ 

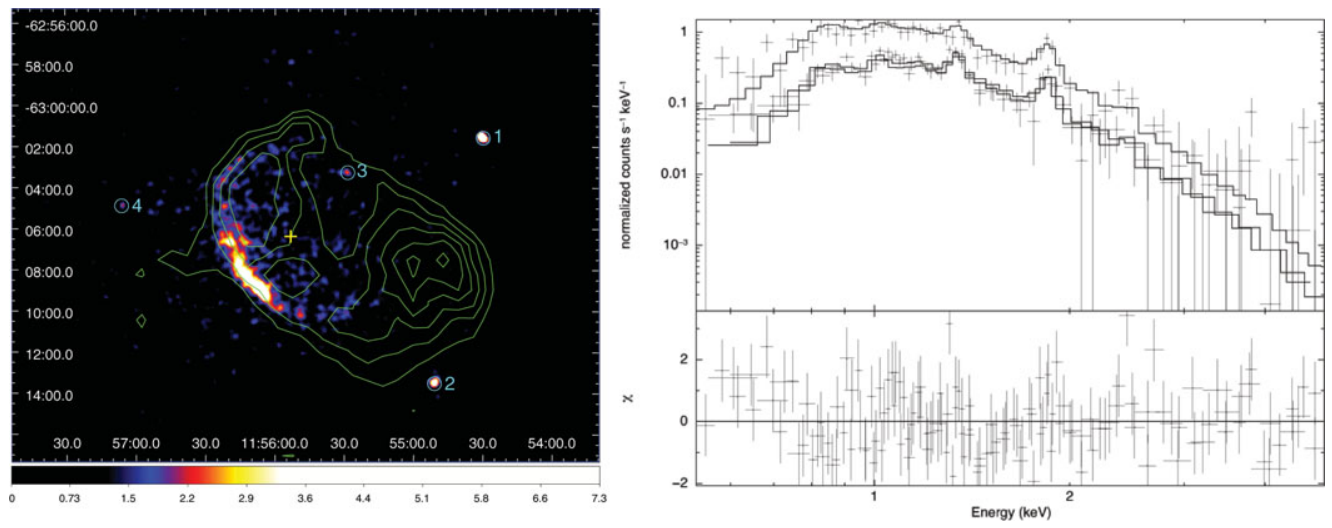

Figure 1. Left: Smoothed XMM-Newton MOS1/2 image of G296.7-0.9 in the energy range of $0.5-10.0 \mathrm{keV}$ with 5-ks exposure time overlaid with the SUMSS radio contour at $843 \mathrm{MHz}$. The geometrical center inferred from the X-ray morphology is illustrated by the yellow cross. The small cyan circles show the positions of 4 X-ray sources. Right: Energy spectrum of G296.7-0.9 obtained from the XMM-Newton MOS1/2 and PN data was fitted with an absorbed CIE model.

full-frame mode using the medium filter to block optical stray light. All the data were processed with the XMM-Newton Science Analysis Software (SAS) package (Version 11.0.0). Calibrated event files for the MOS1, MOS2, and PM detectors were produced using the SAS task emchain and epchain, following standard procedures. In order to remove the particle contamination and periods of high background, we determined a threshold on the light curve counts and then used the SAS task tabgtigen to create the corresponding Good Time Interval (GTI) file. The effective exposure times after background cleaning for MOS1, MOS2, and PN are $4.6 \mathrm{ks}, 5.1 \mathrm{ks}$, and $2.5 \mathrm{ks}$, respectively.

\subsection{Spatial Analysis}

Fig. 1 (left panel) displays the merged XMM-Newton MOS1/2 image of G296.7-0.9 in the energy range of $0.5-10.0 \mathrm{keV}$ with 5-ks exposure time overlaid with the Sydney University Molonglo Sky Survey (SUMSS) radio contour at $843 \mathrm{MHz}$. This image was created by using an adaptive smoothing algorithm with a Gaussian kernel of $\sigma<3$ pixels in order to probe the detailed structure of the diffuse emission. With the better spatial resolution of XMM, an incomplete X-ray shell structure has been revealed. The incomplete X-ray shell-like morphology confirms to an ellipse approximately centered at R.A. $=11^{h} 55^{m} 53^{s}$ and DEC $=-63^{\circ} 06^{\prime} 25^{\prime \prime}(\mathrm{J} 2000)$. The angular sizes of the semi-major and the semi-minor axes are $\sim 4.7^{\prime}$ and $\sim 3.3^{\prime}$, respectively.

In order to search for the possible stellar remnant associated with G296.7-0.9, we used the SAS task edetect_chain. This task runs on the calibrated MOS1/2 and PN image files and invokes several SAS tasks to produce background, sensitivity, and vignettingcorrected exposure maps. In the field of view of the EPIC detectors, 4 sources have been detected. To investigate if these X-ray sources are promising isolated neutron star candidates, we proceeded to search for their possible optical counterparts and calculated the X-ray-to-optical flux ratio $\left(f_{x} / f_{o p t}\right)$, which provides a rudimentary parameter for discriminating the nature of the source. For an isolated neutron star, $f_{x} / f_{\text {opt }}$ is typically larger than 1000 (cf. Haberl 2007) while for field stars and active galactic nuclei the ratio are much lower than the typical $<0.3$ and $<50$, respectively (Maccacaro et al. 1988; Stocke et al. 1991). Based on the X-ray-to-optical flux ratios, no promising isolated neutron star candidate was found in this study. 


\subsection{Spectral Analysis}

We utilized the XMM-Newton SAS tool evselect to extract the spectrum of G296.7-0.9. The background spectrum was extracted from the nearby source-free elliptical region in the same CCD chip. Response files were constructed by using the XMM-Newton SAS tasks rmfgen and arfgen. The spectral modeling was performed with the XSPEC software package (version: 12.7.0) using data in the energy band $0.5-10.0 \mathrm{keV}$.

We examined the spectra with an absorbed collisional ionization equilibrium (CIE) plasma model (XSPEC model: EQUIL) and an absorbed non-equilibrium ionization model of a constant temperature and a single ionization timescale (XSPEC model: NEI). The best-fit CIE model yields a column density of $(1.09 \pm 0.02) \times 10^{22} \mathrm{~cm}^{-2}$ and a plasma temperature of $\mathrm{kT}=0.55_{-0.01}^{+0.02} \mathrm{keV}$ (see Fig. 1 right panel). The ionization timescale and the best-fit plasma temperature inferred from the NEI model are $\tau_{\text {ion }}=\left(6.63_{-2.33}^{+2.95}\right) \times 10^{10} \mathrm{~s} \mathrm{~cm}^{-3}$ and $\mathrm{kT}=0.66 \pm 0.08 \mathrm{keV}$. We found the X-ray spectra of G296.7-0.9 can be described similarly well by either a CIE model or a NEI model. No conclusive evidence for the deviation of metal abundances from the solar values was obtained. Emission lines from various metals can be clearly observed (e.g. $\mathrm{Mg}$ at $\sim 1.4 \mathrm{keV}$; Si at $\sim 1.9 \mathrm{keV}$ ). These properties provide a solid evidence for the hot plasma emission from a remnant. And hence the SNR nature of G296.7-0.9 is confirmed.

We further investigated if there is any non-thermal emission in various regions by adding a power-law model on the aforementioned best-fit plasma models. We found that the parameters of the additional power-law cannot be properly constrained. This prompts us to perform the spectral fit with the photon index fixed at the value of $\Gamma=2$. No significant improvement of the goodness-of-fit was found.

\section{Summary \& Conclusion}

We have performed a detailed spectro-imaging X-ray study of the supernova remnant G296.7-0.9 with XMM-Newton. An incomplete shell-like X-ray structure, which is well correlated with a radio contour, has been revealed. Its X-ray spectrum is thermal dominated and has shown the presence of a hot plasma which has been heated up by shock waves to a temperature of $\sim 6 \times 10^{6} \mathrm{~K}$ and is accompanied with metallic emission lines. All these observational evidences clearly suggest that G296.7-0.9 is a SNR belong to a shell-like category, confirming the previous results reported by Robbins et al. (2012).

\section{References}

Caswell, J. L. \& Haynes, R. F. 1987, A\&A, 171, 261

Fabbiano, G., Kim, D.-W., \& Trinchieri, G. 1994, ApJ, 429, 94

Ferrand, G. \& Safi-Harb, S. 2012, Advances in Space Research, 49, 1313

Green, P. J., et al. 2004, ApJS, 150, 43

Haberl, F. 2007, Ap\&SS, 308, 181

Kuchar, T. A. \& Clark, F. O. 1997, ApJ, 488, 224

Maccacaro, T., Gioia, I. M., Wolter, A., Zamorani, G., \& Stocke, J. T. 1988, ApJ, 326, 680

Manchester, B. A. 1969, Australian Journal of Physics Astrophysical Supplement, 12, 3

Robbins, W. J., Gaensler, B. M., Murphy, T., Reeves, S., \& Green, A. J. 2012, MNRAS, 419, 2623

Schaudel, D. 2003, PhD thesis, Ludwig-Maximilians Universität München

Stocke, J. T., Morris, S. L., Gioia, I. M., Maccacaro, T., Schild, R., Wolter, A., Fleming, T. A., \& Henry, J. P. 1991, ApJS, 76, 813 